\title{
The Applicability of Dispersive and Nondispersive Wave Models for Description of Long Wave Propagation and Run-Up on a Beach
}

\author{
$\underline{\text { Ahmed Abdalazeez }}^{1}$, Denys Dutykh ${ }^{2}$, Petr Denissenko ${ }^{3}$, Ira Didenkulova ${ }^{1,4}$, \\ ${ }^{1}$ Department of Marine Systems, Tallinn University of Technology, Akadeemia tee 15A, Tallinn 12618, Estonia \\ ${ }^{2}$ Univ. Grenoble Alpes, Univ. Savoie Mont Blanc, CNRS, LAMA, 73000 Chambéry, France. \\ ${ }^{3}$ School of Engineering, University of Warwick, Coventry, UK. \\ ${ }^{4}$ Nizhny Novgorod State Technical University n.a. R.E. Alekseev, Minin str. 24, \\ Nizhny Novgorod 603950, Russia.
}

doi: https://doi.org/10.21467/abstracts.93.26

\begin{abstract}
A BS T RA C T
The aim of this work is to study the applicability of dispersive and nondispersive wave models for description of long wave propagation and run-up on a beach in the case ofconstant bottom depth merged with the beach of constant slope. Numerical simulations are performed in the framework of two models: (1) non-dispersive model, based on the Nonlinear Shallow Water (NSW) theory and (2) weakly dispersive model in the Boussinesq approximation, based on the modified Peregrine system. Both models use the finite-volume method with the second-order UNO2 reconstruction in space and the third-order RungeKutta scheme with locally adaptive time steps. Both models also include a Manning friction term to take into account for friction effects on the sloping beach.

The models are compared with experimental data for different types of waves: single waves, sine waves, biharmonic signals and «vessel-like» wave trains, strongly modulated by frequency and amplitude. All used types of waves have the same main characteristic period (20 s) and parameter $k h=0.2$, which corresponds to the shallow water wave conditions (here $\mathrm{k}$ is the wave number and $\mathrm{h}$ is the undisturbed water depth). However, bi-harmonic waves and «vessel-like» wave trains also included waves of shorter periods (10 s), $k h=0.4$. The wave heights are varied in a wide range of values and include both weakly nonlinear and strongly nonlinear (breaking) waves.

Experiments were carried out at the Large Wave Flume, Hannover, Germany (GWK FZK) using the piston type wave maker. The flume has a $251 \mathrm{~m}$ long section of constant depth merged with a plane beach with a slope angle 1:6, made out of rough asphalt, which justifies the inclusion of the friction terms. During experiments with single waves and sine waves, the beach slope was additionally covered by a smooth plastic. The water depth was kept constant at $3.5 \mathrm{~m}$. Wave propagation along the constant bottom section was recorded by 16-18 wave gauges distributed more or less uniformly along the channel.

Shown that for single waves and sine waves, the shallow water theory tends to overestimate the experimental data with a larger error comparing to the weakly dispersive theory. On the other hand, the (weakly) dispersive theory while enjoying a smaller error comparing to the nonlinear shallow water theory, but tends to underestimate slightly the experimentally measured run-up, which makes it a non-safe choice for tsunami hazard estimation. Therefore, for a more accurate forecast we recommend to use the enhanced dispersive theory or the complete hydrodynamic equations. However, for the first rough and "safe" estimate, it is enough to use the classical nonlinear shallow water equations. We would like to stress out the fact that for waves including also shorter components (such as considered bi-harmonic waves and «vessel-like» wave trains), it is the dispersive theory which gives a much more accurate and sensible results.
\end{abstract}

(C) 2020 Copyright held by the author(s). Published by AIJR Publisher in "Abstracts of The Second Eurasian RISK-2020 Conference and Symposium" April 12- 19, 2020, Tbilisi, Georgia. Jointly organized by AMIR Technical Services LLC, Georgian Technical University, Institute of Geography (Kazakhstan) and Russian Institute of Petroleum Geology and Geophysics.

DOI: $10.21467 /$ abstracts. 93 